\title{
Multi-Center Clinical Evaluation of a
}

\section{Chairside Method for Detecting Certain Periodontopathic Bacteria in Periodontal Disease}

\author{
W.J. Loesche, *W.A. Bretz, * D. Lopatin, * J. Stoll,* C.F. Rau, † K.L. Hillenburg, $\dagger$ \\ W.J. Killoy, $\ddagger$ C.L. Drisko, $¥$ R. Williams, H.P. Weber, $\S$ W. Clark, II. Magnusson,\| \\ C. Walker, $\|$ and P.P Hujoel ${ }^{*}$
}

THE ASSOCIATION OF BACTEROIDES GINGIVALIS, Bacteroides forsythus, Treponema denticola, and Actinobacillus actinomycetemcomitans among others with periodontal disease offers the opportunity for the development of diagnostic tests that are based upon the detection and/or quantification of one or more of these organisms or their by-products in the plaque. Three of the putative periodontal pathogens namely, $T$. denticola, $B$. gingivalis, and $B$. forsythus, can hydrolyze the synthetic trypsin substrate, N-benzoylDL-arginine-2-naphthylamide (BANA) forming a color reaction. The present investigation evaluated a commercially developed solid state assay for BANA hydrolysis that can be read after 15 minutes incubation at chairside. A total of 702 subgingival plaque samples were collected from 117 patients seen at four university dental clinics and placed on reagent cards. The color development on the cards was compared to the presence of $T$. denticola and $B$. gingivalis in the plaque, and with the clinical appearance of the sampled sites. This multi-center study demonstrated that antibodies to $B$. gingivalis and $T$. denticola could detect these organisms by an ELISA in the majority of the subgingival plaque samples. Comparable information could be obtained when the same plaques were evaluated by the reagent card format for BANA hydrolysis. The ELISA and reagent card were comparable in their ability to distinguish between clinically healthy and diseased sites. Both diagnostic procedures detected the periodontopathogens in plaques from sites that were judged clinically healthy. The reagent card test has certain clinical advantages over the ELISA in that it is simple, can be performed quickly at chairside, and provides valuable information as to the pathogenicity of the microflora at discrete oral sites. Such information may be used in the detection of patients or intraoral sites at risk for periodontal disease. $J$ Periodontol 1990;61:189-196.

Key Words: Periodontal diseases/diagnosis; periodontal diseases/B. gingivalis; periodontal diseases/B. forsythus; periodontal diseases/Aa; periodontal diseases/Treponema denticola; periodontal diseases/microbiology.

In recent years, persuasive evidence has been presented which suggests a specific bacterial etiology in many forms of periodontal disease. ${ }^{1}$ The association of a limited number of bacterial species such as Bacteroides gingivalis, ${ }^{2,3} \mathrm{Bac}$ teroides forsythus, ${ }^{4,5}$ Wolinella recta, ${ }^{4,5}$ Treponema denticola, ${ }^{6,7}$ and other spirochetes with most forms of adult

\footnotetext{
"University of Michigan School of Dentistry, Ann Arbor, MI.

+University of Detroit School of Dentistry, Detroit, MI.

₹School of Dentistry, University of Missouri at Kansas City, Kansas City, MO.

${ }^{\S}$ Harvard School of Dental Medicine, Boston, MA.

|The University of Florida School of Dentistry, Gainesville, FL.
}

periodontitis and Actinobacillus actinomycetemcomitans with localized juvenile periodontitis and possibly adult periodontitis ${ }^{2,9}$ offers the opportunity for the development of diagnostic tests that are based upon the detection and/or quantification of one or more of these organisms or their by-products, including antigens in the plaque. Indeed, highly specific antibodies ${ }^{7,10-12}$ or flagella stains ${ }^{12}$ and microscopic examinations ${ }^{8}$ have been used to detect some of these organisms in disease-associated plaques. DNA probes to $B$. gingivalis, $A$. actinomycetemcomitans, and Bacteroides intermedius can also be used to detect these species in plaque. ${ }^{13}$ The antibodies and DNA probes, when properly con- 
structed, are so specific that they should detect only the complementary species. Thus if more than one species is suspected as a periodontal pathogen, multiple antibodies, ${ }^{11}$ assay procedures, ${ }^{12}$ and probes ${ }^{13}$ need to be used, thereby increasing the cost of these assays.

All of the suspected periodontal pathogens are Gramnegative organisms and, with the exception of $A$. actinomycetemcomitans, all are anaerobes. Most of these organisms, again with the exception of $A$. actinomycetemcomitans, can use proteins and peptides as nutrients in energy-yielding reactions. These similarities led us to choose metronidazole for use in anaerobic periodontal infections ${ }^{14}$ and to seek a means by which such anaerobic infections could be diagnosed by detecting enzyme activity directed towards proteins and peptides. One such enzyme, namely an enzyme capable of hydrolyzing the synthetic trypsin substrate, $\mathrm{N}$ benzoyl-DL-arginine-2-naphthylamide (BANA), was possessed by $B$. gingivalis, $T$. denticola, and unspeciated Capnocytophaga species ${ }^{15}$ and not by about 15 other plaque species. ${ }^{16}$ Subsequently, it was found that $B$. forsythus, a species associated with the progressive periodontal lesion, also possessed this BANA hydrolytic activity. ${ }^{17}$

Clinical studies showed that subgingival plaques from single sites ${ }^{18}$ or from pooled sites ${ }^{19}$ hydrolyzed BANA and that the presence of this hydrolytic activity could be significantly correlated with probing depths and attachment loss greater than $4 \mathrm{~mm}^{18}$ and with high proportions and numbers of spirochetes in plaque samples. ${ }^{18,19}$ In a subsequent study, BANA hydrolysis by subgingival plaque was evaluated in a blind manner for its ability to reflect both clinical morbidity and subgingival levels of bacteria and spirochetes. ${ }^{20}$ Most BANA positive reactions were from sites which were both clinically diseased and high in spirochetes. The accuracy of the test; i.e., the number of plaques positive for both spirochetes and BANA hydrolysis (true positive) plus the number of plaques negative for spirochetes and BANA hydrolysis (true negative) divided by the total number of plaques samples, was about $94 \%$. Accuracy values for DNA probes for $B$. gingivalis, $B$. intermedius, and $A$. actinomycetemcomitans range from $44 \%$ to $74 \%,{ }^{13}$ whereas monoclonal antibodies for $B$. gingivalis are about $70 \%$ accurate. ${ }^{10}$ These findings indicated that BANA hydrolysis was a simple and accurate indicator of spirochete levels in the plaque and compared favorably with the more expensive DNA probes and immunological reagents.

The present investigation represents an extension of these studies in which the format of the BANA test has been changed from a liquid assay that required overnight incubation to a commercially-developed solid state assay that can be read after 15 minutes incubation at chairside. The purpose of the present investigation was to evaluate the PerioScan format of the BANA test for its ability to detect $T$. denticola and/or $B$. gingivalis in plaque and to determine

؟PerioScan reagent card, Oral-B Laboratories, Redwood City, CA. whether the BANA reaction reflected the presence of clinical disease.

\section{MATERIALS AND METHODS}

\section{Subject Population}

Patients who presented themselves at the Dental Clinics of the University of Detroit, University of Florida, Harvard University, and the University of Missouri at Kansas City were screened for the presence of periodontal disease. Individuals who 1) had at least four teeth radiographically diagnosed as periodontally diseased; 2) had not taken any antibiotics or had periodontal therapy in the last 3 months, and 3) were not currently participants in any other clinical research, were invited to participate in the study. Persons who volunteered signed an informed consent form which described the study and were assigned a subject number from a table of random numbers. Patients were remunerated for participation in the study.

\section{Subject Examinations}

Subject examinations were carried out using an identical protocol, with the exception that at Florida an automatic periodontal probe was used to examine the patients. ${ }^{21}$ One clinician at each site made the clinical evaluations and placed the subgingival plaque samples on the reagent card. Another individual, who was not aware of the clinical evaluations, read and recorded the test results on the reagent cards. The reagent cards from all centers were returned to the University of Michigan where they were screened by an enzyme-linked immunosorbent assay procedure (ELISA) for the presence of $T$. denticola and $B$. gingivalis. Thus the clinical scoring, the reagent card readings, and the immunological determinations were each made by individuals who were unaware of results obtained by the other examiners.

\section{Clinical Examinations}

The clinician at each location selected 12 tooth sites, at least 4 showing radiographic evidence of bone loss and at least 4 showing no evidence of bone loss. In general no more than one site on a given tooth was selected, although on occasion two sites on diametrically opposite sides of the tooth, such as the mid-lingual and mid-buccal of a first molar, were used. Only one of any two adjacent sites in the interproximal space between two teeth was sampled.

A variety of clinical parameters were obtained after the subgingival plaque on the sampled teeth was removed for the BANA test. Gingival health was determined by inserting a wooden toothpick into all interproximal sites and recording the onset and magnitude of bleeding according to the papillary bleeding score (PBS). ${ }^{22}$ Probing depths were determined at 6 locations about the sampled teeth. A Michigan O probe with Williams markings was used at Detroit, Missouri, and Harvard, whereas at Florida a standardized pressure probe ${ }^{21}$ was employed. If bleeding occurred on 
probing (BOP) of the sample site, this was recorded. BOP had been shown to be highly correlated with the presence of spirochetes and $B$. gingivalis in plaque samples. ${ }^{12}$ If the examiner believed that the bleeding was secondary to trauma from plaque sampling, bleeding at the site was scored as questionable. Finally the clinician judged whether the site was healthy or diseased. A healthy site was defined as one in which no root surface debridement was needed above that which would be considered a routine dental cleaning. Healthy sites had a PBS of $\leq 1$, a probing depth $\leq 4 \mathrm{~mm}$, no gingival recession, and no bleeding upon probing. A diseased site was one in which the clinician would recommend some form of root surface debridement. A diseased site had a $P B S \geq 2$, a probing depth $\geq 5 \mathrm{~mm}$, and there was usually bleeding upon probing, whereas there may or may not have been any gingival recession or bone loss.

\section{BANA Test}

The same 12 sites per subject were assigned, using a random number table, to be evaluated by either the liquid BANA test or the solid state reagent card test. Subgingival plaque was removed from each site using a curette and deposited either in a $\mathrm{ml}$ of phosphate buffer for the BANA tests ${ }^{18}$ or directly onto a reagent card. We have not observed any detectable BANA activity remaining on the curette once the visible plaque had been deposited into the buffer, ${ }^{18-20}$ or onto the reagent card. However, as an added precaution, the curette was then wiped carefully with clean gauze before sampling the next tooth. The clinicians were instructed to remove enough plaque per tooth so that it could be visualized on the curette. Such plaque samples weigh at least one mg wet weight and contain about $10^{8}$ bacterial cells. This was done to minimize the likelihood of a false negative result due to the sample size being below the detection limit of the BANA positive species. The vials containing the liquid BANA plaque samples were frozen on dry ice or a freezer at $-10^{\circ} \mathrm{C}$ and sent on dry ice overnight to Michigan where they were thawed, dispersed, examined microscopically for spirochetes, and then divided and assayed by both liquid BANA and the reagent card tests. These findings will be presented in another article.

Each plaque sample used for the reagent card test was deposited in a discrete location on a BANA* impregnated filter strip that ran along the lower border of the card (Fig. 1). An upper reagent strip containing Evans black dye ${ }^{\#}$ was activated by dampening this strip with water. The lower strip was then folded over the upper strip so that any naphthylamide released from the BANA by plaque enzymes would diffuse into the upper strip where it would react with the Evans black dye forming a permanent blue-black color. The two strips were held together by a specially designed clamp. The reagent card held in the clamp was incubated

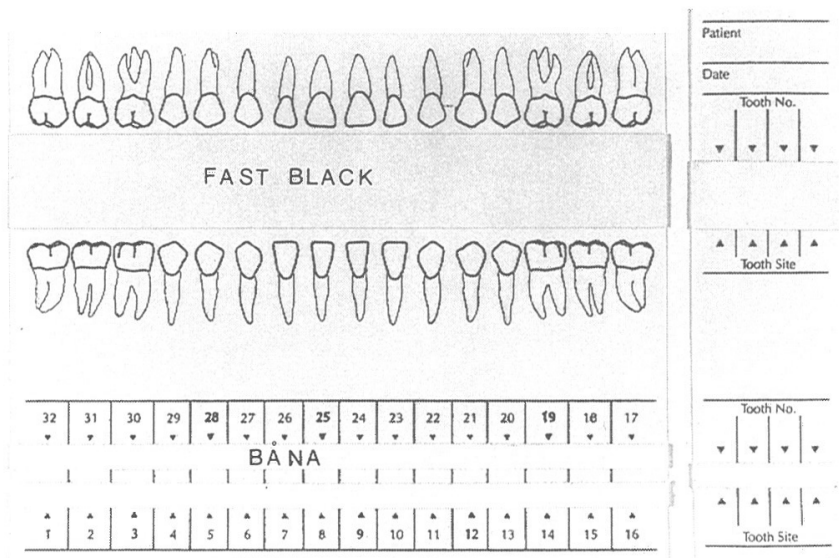

Figure 1: PerioScan reagent card with two thin BANA strips located on the lower border between the tooth numbers and the Fast Black strip located between the tooth drawings. Plaque is placed on the BANA strip adjacent to the tooth number from which the plaque was removed and water is applied to the Fast Black strip. The lower BANA strip is then folded so that it contacts the Fast Black strip and the folded card is held in place by a metal clip. The clip containing the card is then placed within a slot in a heat block and incubated for 15 minutes on a hot plate at $55^{\circ} \mathrm{C}$. The card is removed and any blue-black color present on the Fast Black strip is scored as positive for the tooth adjacent to the color spot.

in a heating block for 15 minutes at about $55^{\circ} \mathrm{C}$, after which time the card was removed from the clamp and the degree of blue-black color was assessed by eye after a training session using the following scale: 0 or negative, no color beyond that of the pink or salmon background color of the reagent strip; 1 or weak positive, a faint blue-black color; 2 or positive, a distinct blue-black color. A faint blue-black color corresponded to about $5 \times 10^{5}$ colony forming units (CFU) of $B$. gingivalis and 1 to $2 \times 10^{6} \mathrm{CFU}$ of $T$. denticola. The color intensity increased with increasing numbers of bacteria. In this report, a weak positive and a positive recording were considered as positive, so that the statistical analysis could be performed on a yes (color) or no (no color) basis.

\section{ELISA}

The lower reagent strip containing the plaque was removed from the reagent card and then the strip was cut in half and each half was stained with highly specific hyperimmune rabbit antibodies to either $B$. gingivalis or $T$. denticola. Antibodies which bound to the plaque were stained with alkaline phosphatase-conjugated goat anti-rabbit Ig. The immune complex was identified and quantitated after incubation with a BCIP/NBT phosphatase substrate system.* This ELISA procedure will detect about $5 \times 10^{4} \mathrm{CFU}$ of either $T$. denticola or $B$. gingivalis. As such, it was about 10 fold more sensitive than the reagent card test in detecting these species. The ELISA reactions were graded as negative or positive. 


\section{Statistics}

The clinical, reagent card, and immunological data were collected from the four centers and entered into a dBaseIII + program. Immunological assays were considered the most reliable procedures currently available for the detection of $B$. gingivalis and $T$. denticola in plaque samples, ${ }^{7,10-12}$ and for this reason an ELISA was chosen as the reference parameter to measure the ability of the reagent card to detect either $B$. gingivalis and/or $T$. denticola.

Since the reference test was not a gold standard, ${ }^{23}$ the terms co-positivity and co-negativity may be preferred over the terms sensitivity and specificity. ${ }^{24}$ However, because the latter terms have been routinely used in statistical analysis in dentistry, we have retained them. These statistical parameters were estimated for each center using the correlated binomial model which allows estimation of sensitivity, specificity, and accuracy, while accounting for the within-patient dependency of site specific results. ${ }^{25}$ The overall measure of sensitivity, specificity, and accuracy across the four centers was taken as a weighted average of the four individual centers, with the weights being the reciprocal of the squared standard errors. For a true positive result, both the reagent card and ELISA tested positive and for a true negative result, both the reagent card and ELISA tested negative. Plaques that were reagent card negative and ELISA positive were considered false negatives, whereas plaques that were reagent card positive and ELISA negative were scored as false positives. The accuracy of the reagent card test was determined by the percentage of co-positive plaques plus the percentage of co-negative plaques as related to the total number of plaques sampled. This is the easiest to interpret of the statistical terms because it tells how often the test parameter agrees with the reference parameter.

We also wanted to determine to what degree both the reagent card test and the ELISA assays for $B$. gingivalis and $T$. denticola agreed with the clinical parameters of periodontal disease; i.e., probing depths, bleeding on probing, papillary bleeding index, and clinical judgement. This agreement was estimated by the overall proportion of agreement of the bacteriological parameters. A jackknifing methodology which accounted for the within-patient correlation of observations ${ }^{26}$ was used to estimate the proportion of agreement and its standard error for each center. The overall measure of agreement across the four centers was taken as a weighted average of the four individual centers, with the weights being the reciprocal of the squared standard errors.

\section{RESULTS}

A total of 117 subjects were examined at the four clinical centers. The age and the sex ratio of the subjects was comparable between the centers, except possibly for Missouri where the patients were older and slightly more males were studied (Table 1). All but one patient had detectable $T$. denticola or $B$. gingivalis as measured by ELISA in at least one of their plaque samples, and 526 of the 702 sampled
Table 1: Characteristics of the Patient Populations at the Four Clinical Centers

\begin{tabular}{lccccc}
\hline Institution & $\begin{array}{c}\text { Number of } \\
\text { Subjects }\end{array}$ & $\begin{array}{c}\text { Mean age } \\
\text { in years }\end{array}$ & Age Range & Females & Males \\
\hline Detroit & 30 & 47.5 & $23-80$ & 19 & 11 \\
Missouri & 30 & 55.2 & $33-77$ & 14 & 16 \\
Harvard & 27 & 45.6 & $25-76$ & 16 & 11 \\
Florida & 30 & 44.3 & $27-74$ & 16 & 14 \\
Four centers & & & & & \\
$\quad$ combined & 117 & 48.0 & $23-80$ & 65 & 52 \\
\hline
\end{tabular}

plaques $(75 \%)$ had one or both of these organisms. The frequency of plaque colonization by these organisms in patients seen at the centers as measured by the ELISA reagents was as follows: Harvard, $62 \%$ of plaques colonized; Detroit, $70 \%$; Missouri, $80 \%$; Florida, $84 \%$.

The relationship between the ELISA results for $T$. denticola and/or $B$. gingivalis and the BANA reagent card reactions for each center is given in Table 2 . The sensitivities in the four centers ranged from $83 \%$ to $95 \%$ with a weighted average of $92 \%$, indicating that when the ELISA was positive for one or both species, the BANA was aimost always positive. The specificity ranged from $53 \%$ to $77 \%$ with a weighted average of $70 \%$, indicating that in the majority of instances in which the ELISA reactions were negative, the BANA reactions were also negative. The accuracy ranged from $79 \%$ to $88 \%$ with a weighted average of $84 \%$.

$B$. gingivalis and $T$. denticola alone or together were detected in about $90 \%$ of the 526 plaques that were reagent card positive. These species were found together in $65 \%$ of these plaques; $T$. denticola alone was found in $10 \%$ of the plaques, and $B$. gingivalis alone was found in $14 \%$ of these plaques (Table 3 ). This left $10 \%$ of the plaques that were reagent card positive, presumably due to the presence of $B$. forsythus or other yet to be identified BANA positive species. B. forsythus and these unidentified species could, however, have contributed to the BANA reaction in those plaques containing $T$. denticola and $B$. gingivalis, and this is indicated by the question mark in Table 3 .

The relationships between colonization of plaques with $T$. denticola and/or $B$. gingivalis as measured by ELISA and several clinical parameters are shown in Table 4. About $89 \%$ of the sites with a probing depth $\geq 5 \mathrm{~mm}$ were colonized by one or both of these organisms (co-positive), whereas only $34 \%$ of the sites $\leq 4 \mathrm{~mm}$ were not colonized by these organisms (co-negative) giving an accuracy value of 55\%. There were few false negatives; i.e., the absence of indicator organisms in deep pockets. The accuracy improved slightly when the comparison involved either $T$. denticola or $B$. gingivalis. However, the high percentages of false positive; i.e., the organisms detectable in shallow pockets, indicated that probing depth was not a suitable reference criterion to evaluate the relative importance of these two organisms in periodontal disease.

When bleeding on probing was used as the reference value, the monitored organisms were present in $83 \%$ of the 
Table 2: Sensitivity, Specificity, and Accuracy of the BANA Reagent Card when Using ELISA Reference Tests Against $T$. denticola and $B$. gingivalis

\begin{tabular}{lccccccccc}
\hline Center & Sensitivity & S.E. $^{*}$ & $\rho \dagger$ & Specificity & S.E. & $\rho$ & Accuracy & S.E. & $\rho$ \\
\hline Detroit & $95 \%$ & $2 \%$ & $\ddagger$ & $68 \%$ & $8 \%$ & $\ddagger$ & $87 \%$ & $3 \%$ & .12 \\
Missouri & $85 \%$ & $3 \%$ & .04 & $53 \%$ & $9 \%$ & .07 & $79 \%$ & $4 \%$ & .07 \\
Harvard & $83 \%$ & $4 \%$ & .17 & $77 \%$ & $5 \%$ & $\ddagger$ & $81 \%$ & $3 \%$ & .00 \\
Florida & $92 \%$ & $3 \%$ & .10 & $67 \%$ & $12 \%$ & .28 & $88 \%$ & $3 \%$ & .17 \\
Weighted & & & & & & & & & \\
Average & $92 \%$ & $1 \%$ & - & $70 \%$ & $4 \%$ & - & $84 \%$ & $2 \%$ & .02 \\
\hline
\end{tabular}

*S.E. = standard error.

$\dagger \rho=$ within-patient correlation coefficient of sites-specific responses.

$\ddagger=$ jackknifing methodology was used to obtain the estimates and their variances because boundary conditions were met by the maximum likelihood estimation methods.

Table 3: Relationship Between ELISA Detection of $T$. denticola and/or B. gingivalis in Plaque and PerioScan Reagent Card Reaction

\begin{tabular}{|c|c|c|c|c|c|}
\hline \multicolumn{3}{|c|}{ BANA Positive Species } & \multirow{2}{*}{$\begin{array}{c}\text { All } \\
\text { Plaques }\end{array}$} & \multicolumn{2}{|c|}{ PerioScan Reagent Card } \\
\hline T. denticola & B. gingivalis & Others* & & Positive plaques & Negative plaques \\
\hline+ & + & $? \dagger$ & $367 \ddagger$ & $340 \div$ & $27 \ddagger$ \\
\hline+ & - & ? & 69 & 55 & 14 \\
\hline - & + & ? & 86 & 72 & 14 \\
\hline - & - & + & 59 & 59 & \\
\hline - & - & - & 121 & & 121 \\
\hline 436 & $\overline{453}$ & $5 \overline{9+} ?$ & 702 & $5 \overline{26}$ & $\overline{176}$ \\
\hline
\end{tabular}

*Others would include $B$. forsythus and other unidentified BANA positive species.

$\dagger$ ? indicates that the presence of other BANA positive species is not known.

$\ddagger$ Number of plaques.

Table 4: Relationship Between ELISA Detection of Bacteroides gingivalis (Bg), and Treponema denticola (Td) Together or Alone and Clinical Parameters

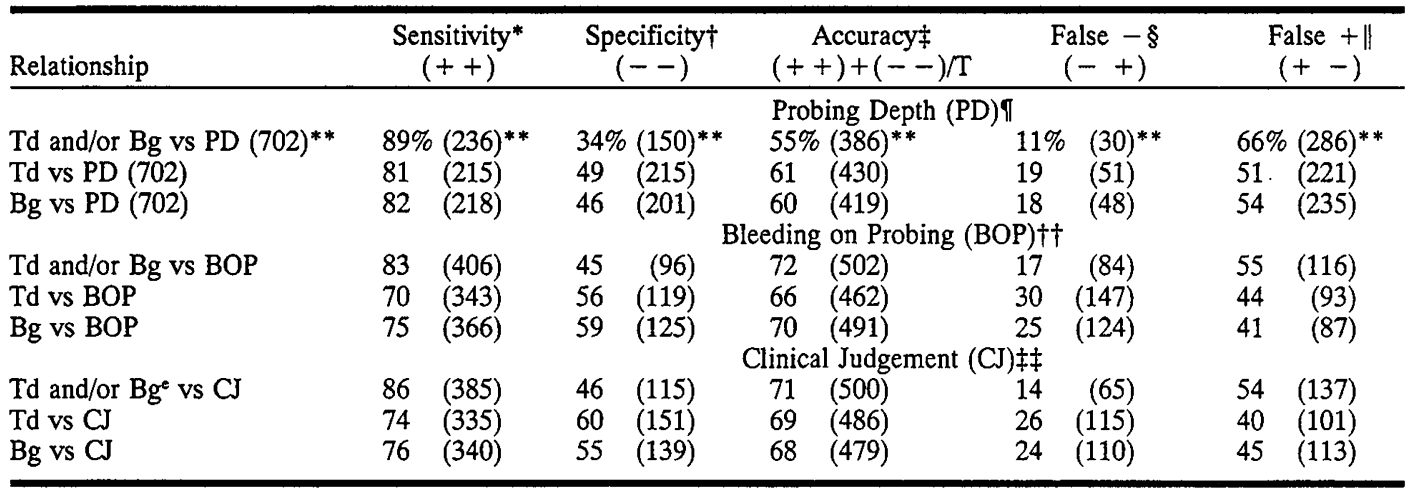

${ }^{*}$ Sensitivity $=\mathrm{Td}$ and/or $\mathrm{Bg}$ positive; clinical parameter positive.

$\dagger$ Specificity $=\mathrm{Td}$ and/or Bg negative; clinical parameter negative

$\ddagger$ Accuracy = Sensitivity plus specificity divided by total (T) number of comparisons.

$\S$ False negative $=\mathrm{Td}$ and/or $\mathrm{Bg}$ negative; clinical parameter positive.

$\|$ False positive $=\mathrm{Td}$ and/or $\mathrm{Bg}$ positive; clinical parameter negative.

ๆProbing Depth; negative is 1 to $4 \mathrm{~mm}$, positive is $\geq 5 \mathrm{~mm}$.

**Number of plaques in parentheses.

††Bleeding on Probing; negative is no; positive is yes.

$\$$ \$Clinical judgement; negative is healthy based on probing depth, BOP, bone height on radiographs and need for debridement; positive is diseased based on probing depth, BOP, bone height on radiographs and need for debridement. See Materials and Methods for added detail.

sites that bled and were absent in $45 \%$ of the sites that did not bleed, giving an accuracy value of about $72 \%$ (Table 4). When the clinicians were forced to judge whether a site needed or did not need some form of root surface debridement, $T$. denticola and/or $B$. gingivalis were found in $86 \%$ of the sites that needed debridement and were absent in
$46 \%$ of the sites that did not need debridement, giving an accuracy value of $71 \%$ (Table 4 ).

We next evaluated the BANA reagent card for its ability to reflect clinical health or disease. The reagent card test was positive in $82 \%$ to $87 \%$ of the comparisons when the clinical parameters indicated 1) disease, 2) that the sites 
BOP, or 3) that the probing depth was $\geq 5 \mathrm{~mm}$ (Table 5). The specificity was about $32 \%$ to $42 \%$, and the accuracy ranged from $53 \%$ to $69 \%$ being best with clinical judgement and BOP.

The ELISA detection of $T$. denticola and $B$. gingivalis in the plaque samples and the reagent card findings were compared for their ability to reflect the clinical parameters employed. The proportion of agreement scores between the clinical judgement and the BANA reagent card was $70 \%$, whereas that between clinical judgement and the ELISA readings was $73 \%$, indicating that the two microbiological parameters were essentially in agreement (Table 6). Comparable agreement scores were obtained when the clinical parameter was BOP or probing depth.

\section{DISCUSSION}

The demonstration that some, if not most, forms of periodontal disease are specific bacterial infections leads logically to the development of diagnostic systems for the detection and/or quantification of these organisms. Several species have been implicated as periodontopathogens, but there is no consensus as to the identification of the exclusive pathogens. There is general agreement that $B$. gingivalis is the most suspect of the black-pigmented bacteroides, ${ }^{2-4}$ that the spirochetes and most likely $T$. denticola are involved, ${ }^{3,6-}$ 8 that $B$. forsythus is associated with the advancing lesion, ${ }^{4,5}$ and that $A$. actinomycetemcomitans is associated with $\mathrm{LJP}^{9,27}$ and other forms of periodontitis. ${ }^{2,11}$ It is not likely that these organisms comprise the entire spectrum of periodontopathogens, but it is apparent that these are the primary organisms for which microbiological diagnostic procedures have been advocated and/or are being developed. ${ }^{10-13}$

All the microbiological diagnostic tests must be evaluated for their ability to detect and possibly quantify these organisms in the plaque using some agreed upon standard. This standard, in the case of the easily cultivable species such as $B$. gingivalis, $B$. intermedius and $A$. actinomycetemcomitans, would seem to be the number of these organisms detected by cultural means. We previously had minimized $B$. gingivalis as being an important contributor to the plaque BANA activity because of its infrequent detection by cultural methods in BANA positive plaque samples. ${ }^{18}$ However, more recent findings using monoclonal and polyclonal antibodies to $B$. gingivalis ${ }^{10}$ or DNA probes to $B$. gingivalis, $B$. intermedius, and $A$. actinomycetemcomitans ${ }^{13}$ indicate that the cultural procedure may underestimate the actual presence of these organisms in the plaques. In fact, when the DNA probes were compared with the cultural as the reference procedure, the false positive value for $A$. actinomycetemcomitans was $39 \%$; the false positive value for $B$. gingivalis was $71 \%$; and the false positive value for $B$. intermedius was $67 \% .^{13}$

This raises a dilemma as to what standard to use in evaluating the reliability and accuracy of a bacteriological di-
Table 5: Relationship Between PerioScan Reagent Card and Clinical Parameters

\begin{tabular}{|c|c|c|c|}
\hline $\begin{array}{l}\text { PerioScan } \\
\text { Reagent Card }\end{array}$ & $\begin{array}{l}\text { Sensitivity* } \\
(++)\end{array}$ & $\begin{array}{l}\text { Specificity* } \\
(--)\end{array}$ & $\begin{array}{c}\text { Accuracy* } \\
(++)+(--) / T\end{array}$ \\
\hline vs CJ† & $84 \%(379)$ & $42 \%(105)$ & $69 \%(484)$ \\
\hline vs $\mathrm{BOP} \ddagger(702)$ & $82 \quad(401)$ & (87) & $70 \quad(488)$ \\
\hline vs $\mathrm{PD} \S(702)$ & $87 \quad(231)$ & (141) & $53 \quad(372)$ \\
\hline
\end{tabular}

${ }^{*}$ Definitions same as in Table 4, except that PerioScan reagent card is substituted for $\mathrm{Bg}$ and/or $\mathrm{Td}$.

$\dagger \mathrm{CJ}=$ Clinical judgement defined as in Table 4.

$\ddagger \mathrm{BOP}=$ Bleeding on probing defined as in Table 4.

$\S P D=$ Probing depth defined as in Table 4.

|number of plaques in parenthesis.

Table 6: Proportion of Agreement Scores Between Clinical Parameters Versus PerioScan ${ }^{\circledR}$ and Elisa

\begin{tabular}{lcccc}
\hline & $\begin{array}{c}\text { Agreement } \\
\text { BANA }\end{array}$ & S.E. & $\begin{array}{c}\text { Agreement } \\
\text { ELISA }\end{array}$ & S.E. \\
\hline CJ & $70 \%$ & $2 \%$ & $73 \%$ & $2 \%$ \\
BOP & $69 \%$ & $2 \%$ & $72 \%$ & $2 \%$ \\
PD & $51 \%$ & $2 \%$ & $55 \%$ & $2 \%$ \\
\hline
\end{tabular}

All estimates were obtained using jackknife estimation procedures taking into account the correlated nature of sites. Scores represent weighted averages of the estimates of the 4 different clinical centers.

See Table 4 for definitions.

agnostic test, if cultural data fail to detect the sought after organism. It has been suggested that immunological procedures should be used as the reference standard for DNA probes $^{13}$ and indeed we have chosen to use immunological reagents to $B$. gingivalis and $T$. denticola as the standard for our BANA reagent card assay. However, the immunological standards are fallible in that cross-reactivity with other species cannot be ruled out, nor is there absolute assurance that all antigenic determinants in the plaque are available for a reaction with the specific antibody. These considerations indicate that not only is there no gold standard to which one can compare new microbiological tests such as DNA probes or the BANA test to, but that the established cultural and immunological procedures, which could be used as references, need themselves to be validated as standards. ${ }^{23}$ Thus, in the discussion which follows it should be recognized that our use of the ELISA reactions was an attempt to validate the BANA results against a standard which we considered to be among the best available for the sought-after BANA positive species and indeed, it may be the only way to detect $T$. denticola, but which falls short of being a gold standard. This point is illustrated by the absence of an antibody to $B$. forsythus which may have explained why the specificity (co-negativity ${ }^{26}$ ) of the BANA test was $70 \%$ (Table 2), whereas the true specificity, if the ELISA reagents to all the BANA positive species were available, most likely would have been higher.

When the reagent card results were compared to the ELISA, a sensitivity of $92 \%$ was found, which provides strong evidence that the reagent card was detecting $B$. gin- 
givalis and/or $T$. denticola in those plaque samples which had these organisms. Thus in $8 \%$ of the plaque samples, the reagent card will fail to detect either $B$. gingivalis and/ or $T$. denticola when they are detectable by the ELISA. This small difference can be explained by the ability of the ELISA to detect as few as $10^{4}$ to $10^{5} \mathrm{CFU}$ of these organisms, whereas the reagent card's detection limits are $5 \times 10^{5}$ to $10^{6} \mathrm{CFU} .^{23}$ Thus, the ELISA will detect lower levels of $T$. denticola and/or $B$. gingivalis that would be missed by the reagent card using the recommended 15 minute incubation time. This could be interpreted as an advantage for the reagent card, as it is not likely to be positive unless the BANA positive organisms comprise $>0.5 \%$ to $1 \%$ of the plaque mass. (If $10^{8} \mathrm{CFU}$ weighs about $1 \mathrm{mg}$, and there is about $1 \mathrm{mg}$ of plaque in a pocket $6 \mathrm{~mm}$ or more, ${ }^{28}$ then 0.5 to $1.0 \%$ of the plaque mass would contain $5 \times 10^{5}$ to $10^{6}$ CFU.)

The specificity of the reagent card relative to the ELISA was $70 \%$, so that the false positives accounted for $30 \%$ of the results (Table 2). This meant that in a high proportion of the plaques the reagent card test was positive while the ELISA was negative for $B$. gingivalis and $T$. denticola. This may be the result of the reagent card test detecting BANAhydrolyzing bacteria other than the $B$. gingivalis and $T$. denticola, such as $B$. forsythus. ${ }^{17}$ As $B$. forsythus can be associated with the advanced periodontal lesion and adult periodontitis, ${ }^{29}$ this would represent an added advantage of the reagent card. If we had possessed an antibody for $B$. forsythus, it is possible that some of these false positive plaques would have been positive for $B$. forsythus and thus would have converted to being true positives.

The accuracy of the microbiological information obtained by the reagent card was comparable to that obtained by the ELISA in $84 \%$ of the plaques (Table 2). When one takes into account the simplicity, quickness; i.e., less than 5 minutes to collect 4 samples and the low cost of the reagent card test; i.e., a projected cost to the clinician of $\$ 0.25$ to $\$ 1.00$ per plaque sample, then it may well be cost efficient relative to the ELISA, if it can also provide the same clinical information as the ELISA with regard to the diagnosis of periodontal infections. The ELISA was able to reflect the presence of infection in $86 \%$ of the diseased sites, whereas the reagent card reflected the presence of infection in $84 \%$ of the diseased sites (see sensitivity for CJ in Tables 4 and $5)$.

The ELISA had a relatively low specificity, or high proportion of false positives, because in 54\% of the healthy sites the ELISA was detecting $B$. gingivalis and/or $T$. denticola. This result can be explained by the ability of the ELISA procedure to detect low levels of these organisms, and doing so at sites in which the bacterial challenge to the gingival tissue from these organisms was either nonexistent or at a subclinical level. Thus the ELISA could be recording colonization of the site and not clinical infection. Alternately, the false positives could be explained by the inherent variability and subjectivity of the clinical judgement, as well as its component parts; i.e., probing depth, bleeding on probing, and the need for treatment. Indeed the ubiquity of these clinical parameters is the main argument in the literature ${ }^{30,31}$ for objectively determined diagnostic tests.

The accuracy of the ELISA in agreeing with the clinician's subjective determination of periodontal health or disease was $71 \%$. This value compares favorably with the accuracy of $69 \%$ obtained by an immunofluorescent assay with a polyclonal antibody to $B$. gingivalis or the accuracy of $72.5 \%$ obtained with a monoclonal antibody to $B$. gingivalis. ${ }^{10}$ An accuracy of about $70 \%$ may reflect what can realistically be achieved by any diagnostic test when it is compared to clinical parameters that are dependent upon probing depths. This is because clinical probing depths themselves are innately highly variable measurements, ${ }^{21,32,33}$ with the magnitude of the variation increasing with probing depth to the extent that perhaps one third of the attachment changes which exceed two population standard deviations are false positive error rates due to measurement error. ${ }^{34}$

The reagent card was able to reflect the presence of clinical disease in about $82 \%$ to $87 \%$ of the sampled clinical sites, depending upon which clinical parameter was used (Table 5). It had a low specificity which could mean that it was detecting colonization of the site, rather than clinically observable infection by these BANA positive species, and in this regard was providing data similar to that of the ELISA. In fact, an examination of Table 6 shows a remarkable agreement between the statistical values obtained either with the ELISA using antibodies to $B$. gingivalis and $T$. denticola, or with the reagent card when both diagnostic parameters were compared to clinical judgement, BOP, and probing depth. Thus, if the ELISA is accepted as the bacteriological standard, and $B$. gingivalis, $T$. denticola and $B$. forsythus are the major periodontopathogens, then the reagent card test yielded results that were comparable to this standard with regard to clinical status. Both tests were detecting periodontopathic organisms in the absence of clinical disease and are thus detecting sites which may be at risk for periodontal disease in susceptible patients. Therefore, it may be prudent to consider treatment plans to insure that these sites do not progress to clinical periodontal disease. These may include improved oral hygiene, heightened professional monitoring, or perhaps subgingival antimicrobial irrigation.

\section{Acknowledgment}

This research was supported by a grant from Oral-B Laboratories, Redwood City, CA. Jennifer Knewitz and Randall Gordon assisted in the study at The University of Missouri at Kansas City. Neal Van Poperin assisted in the ELISA reading, and Carol Gerlach typed the manuscript. 


\section{REFERENCES}

1. Loesche WJ. The bacterial etiology of periodontal disease: The specific plaque hypothesis. In: Clinical Dentistry, JW Clark, ed. Philadelphia: Harper \& Row; Philadelphia 1987.

2. Slots J, Genco RJ. Black-pigmented Bacteroides species, Capnocytophaga species and Actinobacillus actinomycetemcomitans in human periodontal disease: Virulence factors in colonization, survival and tissue destruction. J Dent Res 1984; 63:412.

3. Loesche WJ, Syed SA, Schmidt E, Morrison EC. Bacterial profiles of subgingival plaques in periodontitis. J Periodontol. 1985; 56:447456.

4. Dzink JL, Socransky SS, Haffajee AD. The predominant cultivable microbiota of active and inactive lesions of destructive periodontal diseases. J Clin Periodontol 1988; 15:316-323.

5. Tanner AC, Dzink JL, Ebersole JL, Socransky SS. Wolinella recta, Campylobacter concisus, Bacteroides gracilis, and Eikenella corrodens from periodontal lesions. J Periodont Res 1987; 22:327-330.

6. Moore WEC, Holdeman LV, Cato EP, et al. Comparative bacteriology of juvenile periodontitis. Infect Immun 1985; 48:507-519.

7. Simonson LG, Goodman CH, Bial JJ, Morton HE. Quantitative relationship of Treponema denticola to severity of periodontal disease. Infect Immun 1988; 56:726-728.

8. Loesche WJ. The role of spirochetes in periodontal disease. Advan Dent Res 1988; 2:275-283.

9. Zambon JJ. Actinobacillus actinomycetemcomitans in human periodontal disease. J Clin Periodontol 1985; 12:1-20.

10. Zambon JJ, Reynolds HS, Chen P, Genco RJ. Rapid identification of periodontal pathogens in subgingival dental plaque. Comparison of indirect immunofluorescence microscopy with bacterial culture for detection of Bacteroides gingivalis. J Periodontol 1985; 56(11 Suppl.):32-40.

11. Bragd L, Dahlen G, Wikstrom M, Slots J. The capability of Actinobacillus actinomycetemcomitans, Bacteroides gingivalis and Bacteroides intermedius to indicate progressive periodontitis, a retrospective study. J Clin Periodontol 1987; 14:95-99.

12. Drisko CL, Brandsberg JW, Walters PL, Killoy WJ, Tira DE. Fluorescent antibody and flagella stains for rapid detection of bacteria at periodontally healthy and diseased sites. J Periodontol 1986; 57:542550.

13. Savitt ED, Strzempko MN, Vaccaro KK, Peros WJ, French CK. Comparison of cultural methods and DNA probe analysis for the detection of Actinobacillus actinomycetemcomitans, Bacteroides gingivalis and Bacteroides intermedius in subgingival plaque samples. $J$ Periodontol 1988; 59:431-438.

14. Loesche WJ, Syed SA, Morrison EC, Kerry GA, Higgins T, Stoll J. Metronidazole in periodontitis. I. Clinical and bacteriological results after 15 to 30 weeks. $J$ Periodontol 1984; 55:325-335.

15. Laughon BE, Syed SA, Loesche WJ. API-ZYM system for identification of Bacteroides sp., Capnocytophaga sp. and spirochetes of oral origin. J Clin Microbiol 1982; 15:97-102.

16. Loesche WJ. The identification of bacteria associated with periodontal disease and dental caries by enzymatic methods. Oral Microbiol Immunol 1986; 1:65-70.
17. Tanner ACR, Strzempko MN, Belsky CA, McKinley GA. API-Zym and API-ANADENT reactions of fastidious Gram-negative species. $J$ Clin Microbiol 1985; 22:333-335.

18. Loesche WJ, Syed SA, Stoll J. Trypsin-like activity in subgingival plaque: A diagnostic marker for spirochetes and periodontal disease? J Periodontol 1987; 58:266-273.

19. Bretz WA, Loesche WJ. Characteristics of trypsin-like activity in subgingival plaque samples. $J$ Dent Res 1987; 66:1668-1672.

20. Schmidt EF, Bretz WA, Hutchinson RA, Loesche WJ. Correlation of the hydrolysis of Benzoyl-Arginine Naphthylamide (BANA) by plaque with clinical parameters and subgingival levels of sprirochetes in periodontal patients. $J$ Dent Res 1988; 67:1505-1509.

21. Gibbs CH, Hirschfeld JW, Lee JG, et al. Description and clinical evaluation of a new computerized periodontal probe-the Florida probe. $J$ Clin Periodontol 1988; 15:137.

22. Loesche WJ. Clinical and microbiological aspects of chemotherapeutic agents used according to the specific plaque hypothesis. $J$ Dent Res 1979; 58:2404-2412.

23. Loesche WJ. Hujoel P. Microbiological-based diagnostic tests for periodontitis. Considerations in regard to sensitivity; specificity and accuracy. The Royal Society of Medicine Symposium: Markers of Disease Susceptibility and Activity for Periodontal Diseases. Cambridge, U.K.: Cambridge University Press; in press.

24. Gart JJ, Buck AA. Comparison of a screening test and a reference test in epidemiologic studies. II. A probabilistic model for the comparison of diagnostic tests. Am J Epidemiol 1966; 83:593-602.

25. Hujoel P, Loesche WJ, Moulton L. Estimation of sensitivity and specificity of site-specific diagnostic tests. J Dent Res 1989;68(Spec. Issue):237(Abstr. 444).

26. Gladen B. The use of the jackknife to estimate proportions from toxicological data in the presence of litter effects. J Am Statist Assoc 1979; 74:278-283.

27. Mandell RL, Ebersole JL, Socransky SS. Clinical immunologic and microbiologic features of active disease sites in juvenile periodontitis. $J$ Clin Periodontol 1987; 14534-540.

28. Tanner AC, Goodson JM. Sampling of microorganisms associated with periodontal disease. Oral Microbiol Immunol 1986; 1:15-22.

29. Lai C-H, Listgarten MA, Shirakawa M, Slots J. Bacteroides forsythus in adult periodontitis. Oral Microbiol Immunol 1987; 2:152-157.

30. Polson AM, Goodson JM. Periodontal diagnosis. Current status and future needs. J Periodontol 1985; 56:25-34.

31. Listgarten MA. A perspective on periodontal diagnosis. J Clin Periodontol 1986; 13:175-181.

32. Goodson JM. Clinical measurements of periodontitis. J Clin Periodontol 1986; 13:446-460.

33. Cohen ME, Ralls SA. False positive rates in the determination of changes in probing depth related periodontal measurements. $J$ Periodont Res 1988; 23:161-165.

34. Gunsolley JC, Best AM. Change in attachment level. J Periodontol $1988 ; 59: 450$.

Send reprint requests to: Dr. Walter J. Loesche, The University of Michigan, School of Dentistry, Ann Arbor, MI, 48109-1078. Accepted for publication September 25, 1989. 